\title{
Microsurgery for vestibular schwannoma after Gamma Knife surgery: challenges and treatment strategies
}

\author{
Clinical article
}

\author{
Cheng-Chia Lee, M.D., ${ }^{1-3,5}$ Hsiu-Mei Wu, M.D. ${ }^{3,4}$ Wen-Yuh Chung, M.D., ${ }^{1,3}$ \\ Ching-Jen Chen, M.D., ${ }^{5}$ David Hung-Chi Pan, M.D., ${ }^{1,3}$ and Sanford P. C. Hsu, M.D. ${ }^{1,3}$
}

${ }^{I}$ Department of Neurosurgery, Neurological Institute, and ${ }^{4}$ Department of Radiology, Taipei Veterans General Hospital; ${ }^{3}$ School of Medicine, National Yang-Ming University, Taipei; ${ }^{2}$ Department of Surgery, Hsinchu Branch, Taipei Veterans General Hospital, Hsinchu, Taiwan, Republic of China; and ${ }^{5}$ Department of Neurological Surgery, University of Virginia Health System, Charlottesville, Virginia

\begin{abstract}
Object. Resection of vestibular schwannoma (VS) after Gamma Knife surgery (GKS) is infrequently performed. The goals of this study were to analyze and discuss the neurological outcomes and technical challenges of VS resection and to explore strategies for treating tumors that progress after GKS.

Methods. In total, 708 patients with VS underwent GKS between 1993 and 2012 at Taipei Veterans General Hospital. The post-GKS clinical courses, neurological presentations, and radiological changes in these patients were analyzed. Six hundred patients with imaging follow-up of at least 1 year after GKS treatment were included in this study.

Results. Thirteen patients (2.2\%) underwent microsurgery on average 36.8 months (range 3-107 months) after GKS. The indications for the surgery included symptomatic adverse radiation effects (in 4 patients), tumor progression (in 6), and cyst development (in 3). No morbidity or death as a result of the surgery was observed. At the last follow-up evaluation, all patients, except 1 patient with a malignant tumor, had stable or near-normal facial function.

Conclusions. For the few VS cases that require resection after radiosurgery, maximal tumor resection can be achieved with modern skull-based techniques and refined neuromonitoring without affecting facial nerve function. (http://thejns.org/doi/abs/10.3171/2014.8.GKS141312)
\end{abstract}

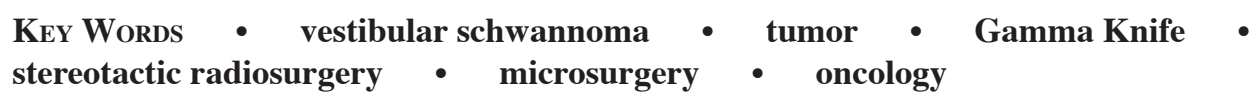

$\mathrm{S}$ TEREOTACTIC radiosurgery (SRS) is now the most commonly used treatment modality for vestibular schwannoma (VS). ${ }^{19}$ Modern SRS series have consistently observed rates of tumor control and facial nerve function preservation of $>90 \%$ in follow-up periods ranging from 2.6 to 12.5 years. ${ }^{6,9,10,14,22,24,33}$ Rates of hearing preservation are moderately lower, ranging from $58 \%$ to $75 \%$ in SRS series with follow-up periods of up to 9.3 years. ${ }^{1,3,6,22,24,33,41}$ Although SRS offers high rates of tumor control and preservation of facial nerve function in patients with VS, radiosurgical treatment fails for a significant subset of patients who subsequently require microsurgical resection.

Our previous report in 2005 demonstrated tumor control and hearing preservation rates of $96.8 \%$ and $60 \%$, re-

Abbreviations used in this paper: $\mathrm{ARE}=$ adverse radiation effect; EMG = electromyography; GKS = Gamma Knife surgery; NF = neurofibromatosis; SRS = stereotactic radiosurgery; VP = ventriculoperitoneal; VS = vestibular schwannoma. spectively, and a low morbidity rate and no death in a cohort of 195 patients with VS treated using Gamma Knife surgery (GKS). ${ }^{7}$ Our subsequent study in 2010 identified 7 patients who required microsurgical resection of their VS after GKS for reasons that included inadequate tumor control and adverse side effects during a 16-year period between March 1993 and December 2008. ${ }^{20}$ To further analyze this subset of patients, we here updated the previous case series with 6 additional patients who underwent microsurgical resection of their VS between December 2008 and December 2012. The goals of this study were to discuss the neurological outcomes and technical challenges of VS resection, and to explore treatment strategies for tumor progression after GKS.

\section{Methods}

This study was a retrospective review of patient records prospectively collected in a Gamma Knife database at the Taipei Veterans General Hospital. Between 1993 
and 2012, 708 patients with VS were treated with GKS at our institution. Of these, 600 with imaging follow-up of at least 1 year after GKS treatment were included for analysis. The demographic data collected included patient sex and age at the time of treatment, and data collected on VS characteristics included tumor volume, tumor lateralization, and histological diagnosis. The radiosurgical data included margin/maximum dose, isodose line, and treatment volume. Patients' pretreatment and posttreatment symptoms, neurological deficits, or both were also included. Imaging studies were obtained for all patients, using standard posterior fossa MRI sequences at our hospital. Precontrast sequences included axial T1-weighted (3-mm sections) and fast spin echo axial T2-weighted (3-mm sections) images. Postcontrast sequences included axial, coronal, and sagittal T1-weighted (3-mm sections) images. Since 2010, imaging sequences acquired with the FIESTA method were used as part of our standard radiosurgical planning.

On the basis of treatment history, patients were categorized into 2 groups: Group A consisted of patients who did not have any microsurgical resection during the follow-up period after GKS, and Group B of patients who underwent microsurgical resection for their VS after GKS. For patients in Group B, detailed information regarding their management were collected, including the length of the time interval between GKS and microsurgery, degree of resection, and sequence of therapies. Outcomes of facial nerve and cochlear nerve functions for these patients were evaluated using the House-Brackmann score at 6 months postoperatively and the GardnerRobertson class at 6 months postoperatively, respectively.

\section{Patient Characteristics}

Of the 600 patients included in this study, 587 did not undergo microsurgical resection of their VS during the follow-up period (Group A), and 13 (2.2\%) underwent microsurgical resection after GKS for reasons that included uncontrolled tumor growth and neurological deterioration (Group B). As shown in Table 1, the median age of the patient in Groups A and B was 51 and 44 years, respectively, and the proportions of females in Groups A and $\mathrm{B}$ were $58.1 \%$ and $76.9 \%$, respectively. The median tumor volume was $4.2 \mathrm{~cm}^{3}$ in Group A and $6.5 \mathrm{~cm}^{3}$ in Group B. Of the patients in Group A, 330, 198, and 59 had tumor volumes of $<3,3-10$, and $>10 \mathrm{~cm}^{3}$, respectively. In Group B, 2, 6, and 5 patients had tumor volumes of $<3$, $3-10$, and $>10 \mathrm{~cm}^{3}$, respectively.

\section{Gamma Knife Surgery}

Radiosurgery was performed using the Leksell Gamma Knife model B between 1993 and 2006, and model C (both Elekta) thereafter. For all patients in Group A, radiosurgery was delivered as a single-session treatment to the tumor, using a median margin dose of $12 \mathrm{~Gy}$ (range 11.0-18.2 Gy). A high margin dose ( $\geq 16 \mathrm{~Gy}$ ) was used infrequently and was applied only to those treated during the early part of this study. The treatment dose was prescribed to a median isodose line of $57 \%$ (range $50 \%-$ $94 \%$ ), and the median maximum dose was $21.6 \mathrm{~Gy}$ (range
17.1-34.0 Gy). Any identifiable portion of the facial nerve received no more than $13 \mathrm{~Gy}$, and the trigeminal nerve received no more than $15 \mathrm{~Gy}$.

Only 1 patient in Group B received a high margin dose of $16 \mathrm{~Gy}$, and the other 12 patients received a median margin dose of $12 \mathrm{~Gy}$ (range 11-12.5 Gy). Treatment dose was prescribed to a median isodose line of 56\% (range $50 \%-60 \%$ ). Radiosurgical data for Group B are shown in Table 2.

\section{Follow-Up Imaging and Clinical Evaluation}

All patients were evaluated clinically and radiographically, using MRI at regular 6-month intervals after GKS. The tumor volume was derived from the sum of the product of the tumor surface area on each MRI section and the thickness of each MRI section $(3 \mathrm{~mm}){ }^{7,8,36,38}$ On the basis of volumetric measurements, the size of the tumor was considered stable if the tumor was approximately $50 \%$ of its original volume, enlarged if it was greater than $50 \%$, or regressed if it was $50 \%$ smaller than its original volume. ${ }^{20}$

Sequential MRI follow-up studies were reviewed, and a certain degree of T2-weighted signal change was observed around the VS. The signal change on T2-weighted images was interpreted as radiation-induced edema, an adverse radiation event (ARE). According to the severity of the edema, the ARE was classified as mild (a narrow rim surround the VS), moderate (less than one-fourth of the brainstem volume involved), or severe (more than one-fourth of the brainstem volume involved). ${ }^{28}$

\section{Resection of Radiated VS}

Patients in Group B underwent microsurgical resection of their VS because of neurological deterioration and inadequate tumor control after GKS. All patients who had deteriorating clinical symptoms and neurological deficits were initially treated using oral prednisolone 5-20 mg daily. Patients whose symptoms did not resolve received intravenous injections of $5 \mathrm{mg}$ dexamethasone 2-4 times a day. Magnetic resonance imaging examinations were subsequently performed, and scans were reviewed by members of the Gamma Knife team.

Patients who underwent microsurgical resection of their VS were placed in either a supine-lateral or a parkbench position. Retrosigmoid craniotomy was performed using a high-speed drill, and the anterior margin of the bone flap on the ipsilateral side was extended to the sigmoid sinus. The durotomy was performed in a C-shaped or radiating manner, and the brain was decompressed by opening the cisterna magna and cerebellopontine cistern. After arachnoid dissection, critical neural and vascular structures were identified, including the lower cranial nerves, petrosal vein, trigeminal nerve, and anterior inferior and posterior inferior cerebellar arteries. Using a facial nerve stimulator, the posterior aspect of the tumor was carefully inspected to confirm the location of the facial nerve. The posterolateral capsule of the tumor was breached using cotton pads, and internal decompression performed using a Cavitron ultrasonic surgical aspirator or a bipolar cautery with scissors. The tumor was col- 
TABLE 1: Characteristics of the 600 patients in this study*

\begin{tabular}{lccc}
\hline \multicolumn{1}{c}{ Parameter } & Group A (no MS after GKS) & Group B (MS after GKS) & p Value† \\
\hline no. of patients & 587 & 13 & 0.273 \\
median age in yrs (range) & $51(10-85)$ & $44(19-70)$ & 0.172 \\
female sex (\%) & $341(58.1)$ & $10(76.9)$ & 0.056 \\
median tumor vol in cm ${ }^{3}$ (range) & $4.2(0.04-25.9)$ & $6.5(2.3-23.5)$ & \\
tumor vol at GKS (\%) & $330(56.2)$ & $2(15.4)$ & 0.976 \\
$<3 \mathrm{~cm}^{3}$ & $198(33.7)$ & $6(46.2)$ & 0.003 \\
$3-10 \mathrm{~cm}^{3}$ & $59(10.1)$ & $5(38.5)$ & 0.003 \\
$>10 \mathrm{~cm}^{3}$ & $12(10-16)$ & $6(11-16)$ & 0.017 \\
median margin dose in Gy (range) & $470(80.1)$ & $7(53.8)$ & NA \\
no. of regressed or stable tumors at FU (\%) & $117(19.9)$ & $71.3(13.4-205.5)$ & $36.8(3.0-107.0)$ \\
no. of progressed tumors at FU (\%) & $49.5(6.0-191.6)$ & NA & \\
median clinical FU in mos (range) & &
\end{tabular}

lapsed inward, and the facial nerve was identified and meticulously dissected away from the tumor capsule.

\section{Monitoring and Mapping}

Before positioning of the patient, the subdermal needle electrodes (12 mm long and 27-gauge diameter; VIASYS) were inserted into the stimulating sites and the corresponding recording sites. The neuromonitoring of the cerebellopontine angle tumors included motor, somatosensory, and brainstem auditory evoked potentials; free-run electromyography (EMG); triggered EMG; and blink reflex. The stimulation of motor evoked potentials was to activate the primary motor cortex, and the subdermal needle electrodes were placed at $\mathrm{C} 1$ and $\mathrm{C} 2$ on the scalp.

For the recordings, subdermal needle electrodes were placed in the muscles of interest: the orbicularis oris and orbicularis oculi (for the facial nerve), soft palate (for the vagus nerve and glossopharyngeal nerve), tongue (for the hypoglossal nerve), and abductor pollicis brevis (for the corticospinal tract). The stimulating electrodes for obtaining somatosensory evoked potentials were usually placed at the ankle for the posterior tibial nerve and at the wrist for the median nerve. The recording sites were on the scalp, corresponding to the parietal cortex. Thus, needle electrodes were placed on Fz, Cp3, and Cp4. Ideally, for the patients who had servable hearing, the stimulation of brainstem auditory evoked potentials was a click, produced by delivering a square wave electrical pulse to a transducer. A plastic tube delivered a sound to the auditory canal of the stimulated ear. We used disposable foam ear tips (XLTEK), one for each ear and connected via a front tube to a transducer. The recording electrodes were subdermal needle electrodes, placed in the ear lobes (A1 and A2) and at Cz.

During the tumor dissection, functional mapping was also performed by triggered EMG. Triggered EMG applies an electrical stimulus directly on the motor nuclei or nerves to elicit compound muscle action potentials recorded in the corresponding muscle channels. Direct nerve bi- and monopolar (VIASYS) stimulation was used for the cranial nerves and for the nuclei. Cranial nuclei were stimulated by delivering a constant current, with repetitive square wave pulses of 0.02-0.05 seconds and with an intensity of 5-8 mA, for an average of 4-6 trials. In addition, the responses of the stimulated pharyngeal, glossopharyngeal, and vagus nerves were recorded with repetitive square wave pulses of $0.1-0.2$ seconds, intensity $0.1-2 \mathrm{~mA}$, and a frequency of $4 \mathrm{~Hz}$, for an average of 4-6 trials.

The motor, somatosensory, and brainstem auditory evoked potentials, and the blink reflex were recorded throughout the operative course. A repeated facial nerve EMG check was always performed when we encountered a radiated VS with severe adhesion and vague anatomic structures. (Fig. 1)

\section{Results}

\section{Changes in Tumor Volume After GKS}

The efficacy of radiosurgery was assessed by examining changes in tumor volume in all 600 patients who underwent regular MRI follow-ups. In Group A, 470 patients $(80.1 \%)$ showed decreased or stable tumor volume at the last follow-up, and 117 (19.9\%) showed an increased tumor volume. The increased volume was mostly found in the early follow-up period, that is, within 6 to 18 months. The median follow-up time for patients in Group A was 49.5 months after the GKS. In Group B, 7 patients (53.8\%) showed an increased tumor volume at the followup (an example is shown in Fig. 2). The median follow-up time for this group was 71.3 months after GKS.

\section{Resection of VS After GKS}

In this study, 13 patients underwent microsurgical re- 


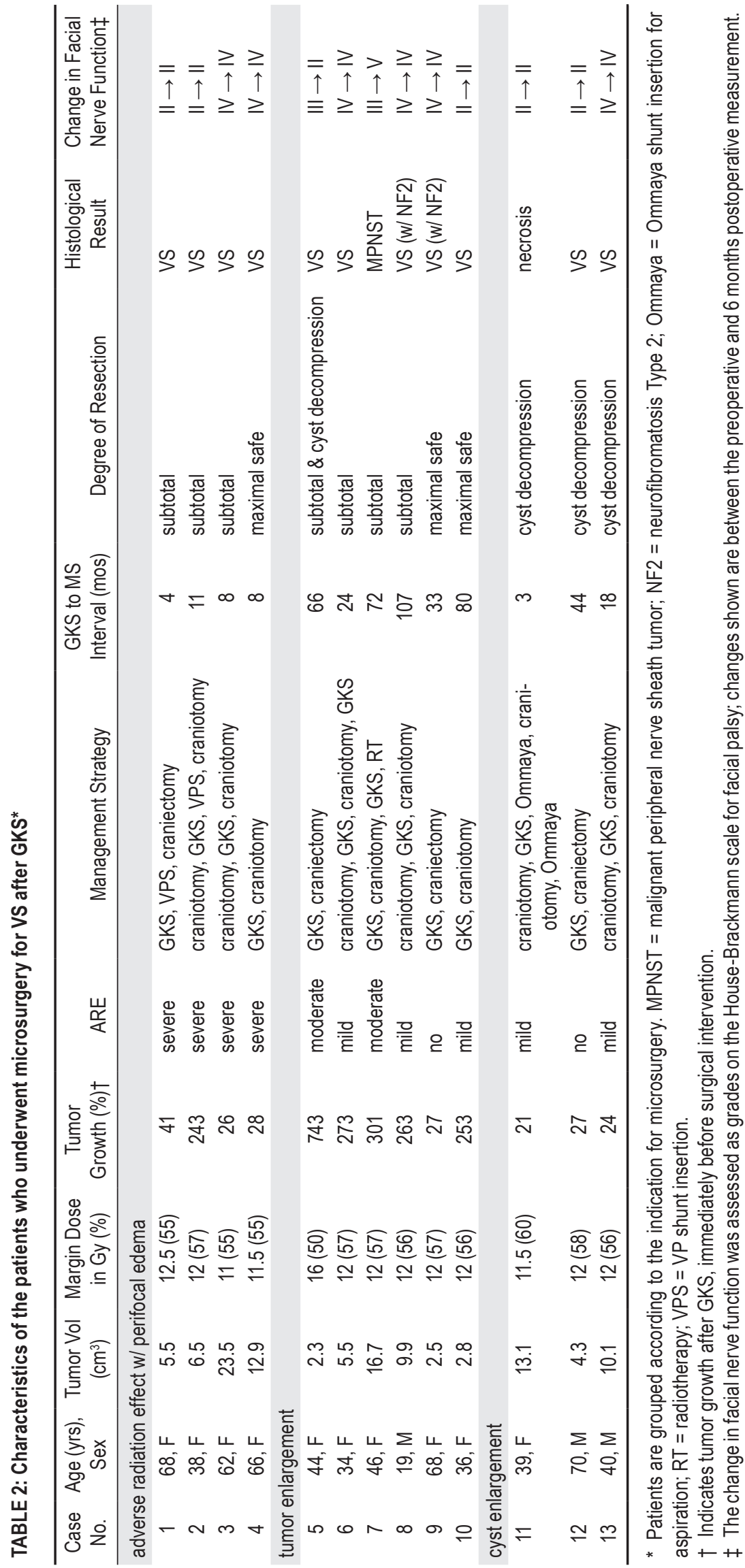


C. C. Lee et al.
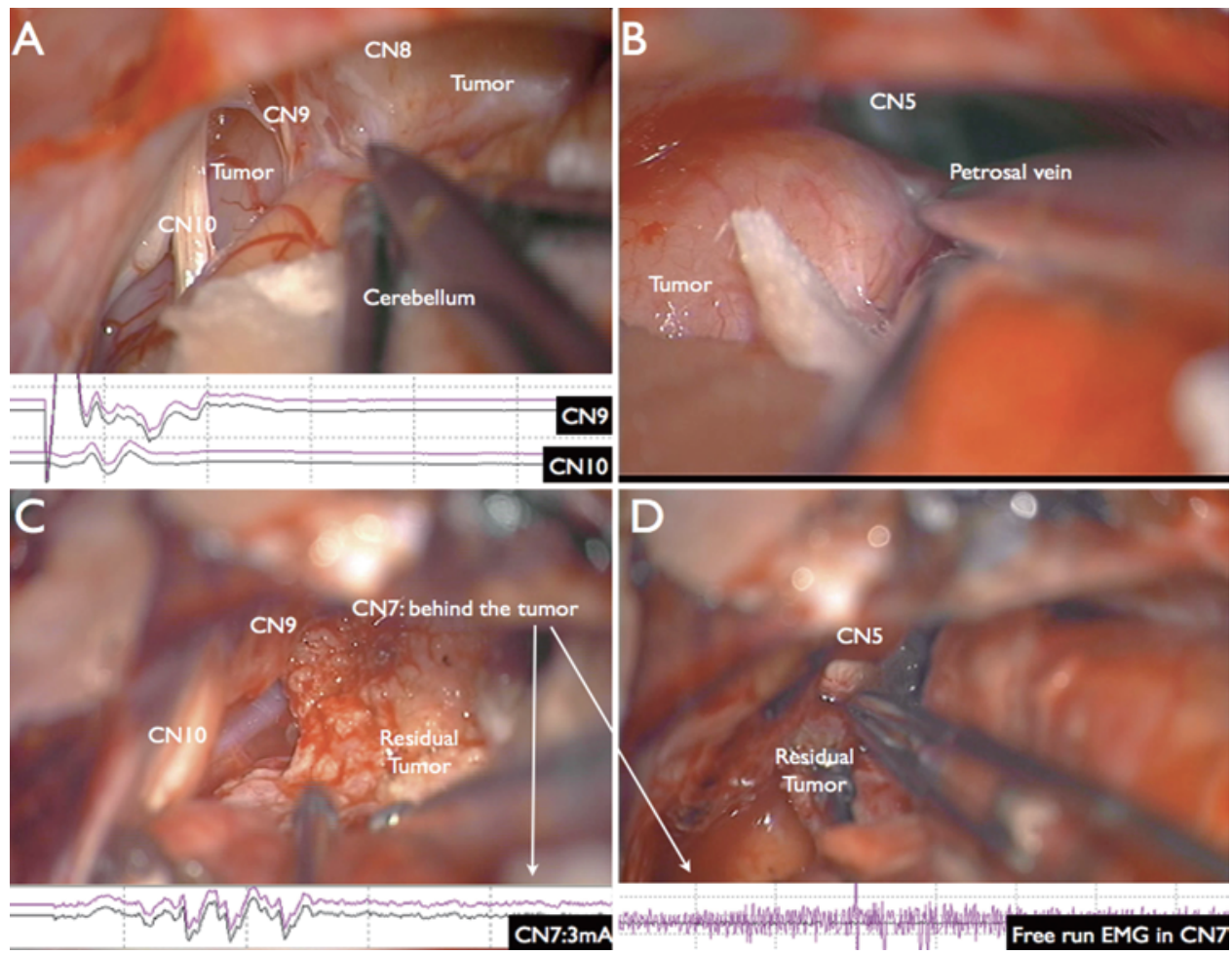

FIG. 1. Intraoperative pictures showing the skull base during tumor resection and the neuromonitoring techniques applied. Details about the patient are described in Fig 2. A: The lower part of the tumor adhered to the nearby cranial nerves, including cranial nerves (CNs) VIII, IX, and X. B: The upper part of the tumor transposed the petrosal vein upward. Note that a fixed retractor was not used. C: A bandlike tumor capsule was left overlying the facial nerve, which was delineated by repeated electric stimulation following identification of the flocculus and choroid plexus. D: During the tumor dissection near CN VII, the free-run electromyogram showed significant firing of the facial nerve.

section after the GKS (Table 1). The mean length of time from the radiosurgery to microsurgery was 36.8 months (range 3-107 months). The 13 patients in Group B tended to harbor larger tumor volumes at GKS than those in Group A (4.2 vs $\left.6.5 \mathrm{~cm}^{3}, \mathrm{p}=0.056\right)$, although the tumors had received a similar margin dose (12 Gy, $\mathrm{p}=0.976)$. Following GKS, the tumor volume had increased on average by $95 \%$ (range $21 \%-743 \%$ ) in these 13 patients. In 3 patients (Cases 11, 12, and 13), the solid components of the tumors increased by approximately $20 \%$. However,

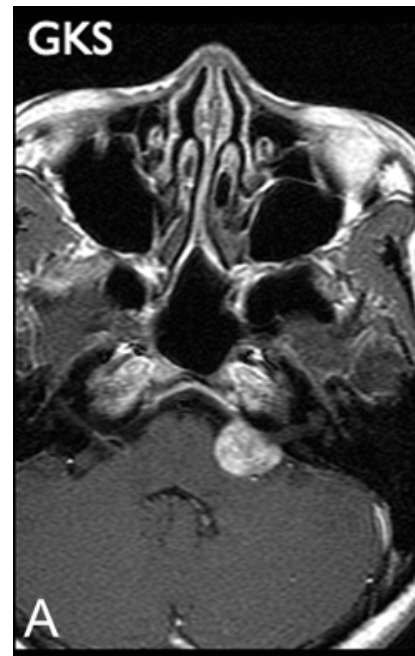

TV: $2.8 \mathrm{ml}$

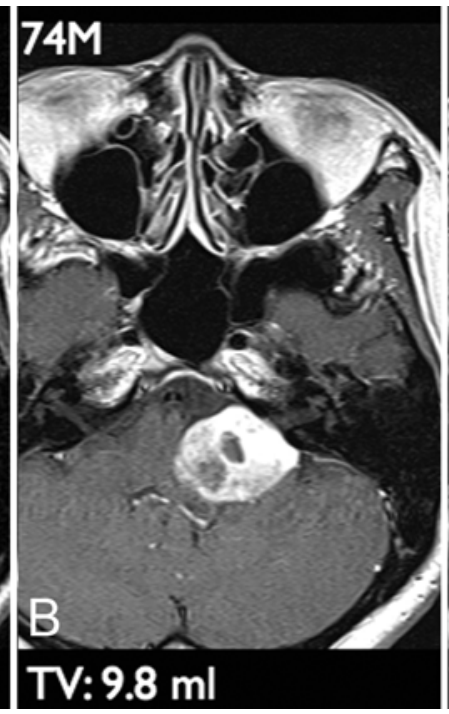

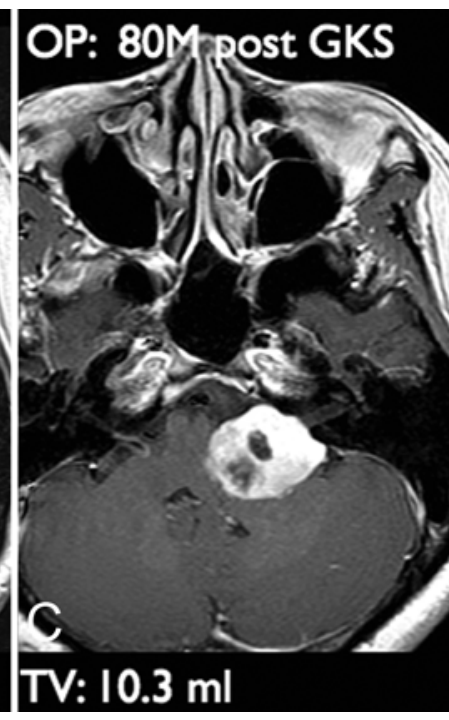

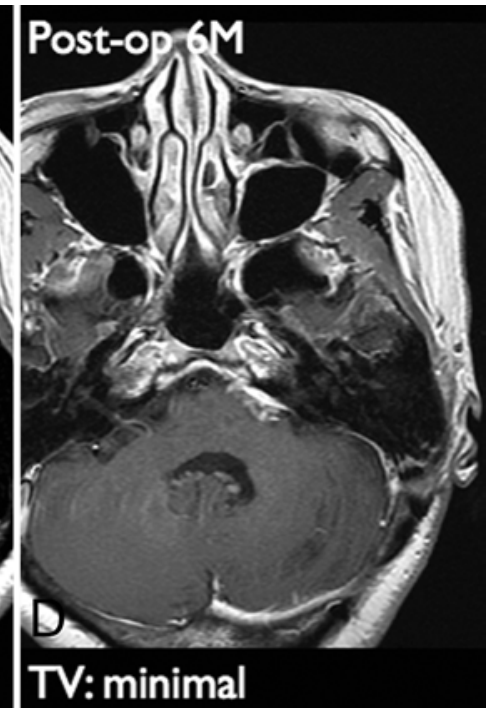

FIG. 2. A-D: Serial axial MRI scans showing the typical appearance of tumor recurrence after a long period of stability. A 36-year-old woman with a 2.8- $\mathrm{cm}^{3}$ VS underwent GKS in 2004. Seventy-four months later, the tumor had grown to $253 \%$ of its original size. Maximal safe resection was achieved, with only a small residual tumor remaining. $M=$ month; $O P=$ operation; TV $=$ tumor volume 
their cystic components enlarged by more than $300 \%$, resulting in a mass effect. Prior to microsurgery, all 13 patients had progressive neurological deteriorations, such as gait ataxia, weakness, or consciousness disturbance. Follow-up MRI scans showed AREs in 4 patients, tumor enlargement in 6, and cyst growth in 3. One patient (Case 2) had both an enlarged tumor and AREs. Seven of these patients had a subtotal tumor resection, 3 a maximal safe resection, and 3 a decompressive drainage of multilobular cysts. The results of the histological analyses of the tumors indicated that 11 patients had a typical benign VS, 1 a malignant peripheral nerve sheath tumor, and 1 a necrotic tissue (Table 2).

As shown in Table 2, we classified the 13 patients in Group B into 3 subgroups on the basis of their surgical indications. Four patients had AREs characterized by tumor swelling and perifocal edema after GKS. In these patients, the time interval between GKS and microsurgery ranged from 4 to 11 months. Although in 3 of these patients the enlargement in tumor volume was less than $50 \%$, the perifocal edema led to significant neurological deterioration. The microsurgery was performed to decompress the brain edema and to immediately resolve this neurological deterioration. The histological analysis of the tumors showed typical benign VS in the 4 patients of this subgroup.

Abnormal tumor enlargement after GKS was the second surgical indication, which was present in 6 of the Group B patients. In these patients, the interval between GKS and microsurgery ranged from 24 to 107 months. Significant tumor growth was observed in all them, with on average a 3-fold increase in tumor volume after a stable period (Fig. 2). The goal of the microsurgery in these patients was not only to decompress, but also to biopsy the tumors to examine them for malignant transformation. The histological examination of the tumors indicated typical benign VS in 5 patients and a malignant tumor of the peripheral nerve sheath in 1 patient.

The last surgical indication was cystic enlargement, which was observed in 3 patients after GKS. The size of the solid component of the tumors did not increase significantly (the increase was $21 \%$ at 3 months, $24 \%$ at 18 months, and $27 \%$ at 44 months after GKS), but the cystic component showed significant enlargement (that is, a mean enlargement of $342 \%$ ), with a mass effect several months after the GKS. One patient (Case 11) with an enlarged cystic component underwent an Ommaya shunting procedure for a multilobular cyst, followed by simple microsurgical aspiration and biopsy 5 months after GKS. Histological examination of the surgical specimen indicated only necrotic tissue, with no active tumor cells. The remaining 2 patients (Cases 12 and 13) in this subgroup received extensive fenestration of a cyst, and the specimen were identified as typical benign VS.

\section{Functional Outcomes}

After microsurgery, 12 patients whose histological results indicated a benign tumor or necrosis showed improvement of symptoms. Further follow-ups with MRI showed stability or reduction in the size of the residual tumor. These 12 patients maintained stable clinical func- tioning (including facial function) for at least a median follow-up time of 3 years after the microsurgery (Table 2). All 12 patients experienced ipsilateral hearing loss preoperatively. Although no major complications were observed after microsurgery, dizziness and tinnitus continued to affect most of the patients.

The patient who had a malignant tumor of the peripheral nerve sheath experienced rapid tumor progression, coinciding with severe ataxia, complete facial palsy, and perturbed consciousness 1 month after the microsurgery. A repeat microsurgical resection, followed by repeat GKS and adjuvant radiotherapy, was performed in attempts to control the tumor. However, significant declines in the patient's neurological functional status (such as complete facial palsy, multiple cranial nerve dysfunctions, and hemiparesis) led to severe disability. This patient died 110 months after the initial GKS.

\section{Discussion}

Tumor control with GKS for small- or medium-sized $(<3 \mathrm{~cm})$ VS results in favorable outcomes, but few studies have reported the outcomes in those patients whose tumor progressed after the GKS treatment. In some instances, tumor progression, cystic expansion, or symptomatic AREs may necessitate subsequent microsurgery or other decompressive procedures. However, the indications and timing of these surgical interventions remain undetermined and controversial because they are rarely performed after GKS. In addition, the long-term outcomes, such as functional preservation or tumor recurrence, of microsurgery after GKS for VS are largely unknown. Given the paucity of data on the management of recurrent VS after GKS, we propose here an algorithm for managing these challenging tumors (Fig. 3).

\section{Indications and Timing of Post-GKS Microsurgery}

Microsurgery may be indicated when patients have progressive neurological deteriorations with 1) symptomatic ARE; 2) tumor progression, whether due to recurrence or a malignant transformation; or 3) cyst formation or enlargement. Proper selection of patients requires a careful review of serial MRI follow-up scans, good knowledge of the disease course and of the pathophysiology after radiosurgery (e.g., of ARE and postradiosurgical tumor expansion), and close observation of clinical symptoms.

Adverse radiation effects or radiation-induced cranial neuropathies now occur in fewer than $10 \%$ of patients after SRS and are usually associated with a tumor size of $3 \mathrm{~cm}$ or larger, with a higher tumor margin dose, or both. ${ }^{32}$ The AREs are most commonly encountered 6-18 months after radiosurgery and in more than half of the cases, resolve 3-6 months after onset.,32,36 We suggest avoiding surgery during these periods if possible, since the cranial nerves and brainstem are most vulnerable to secondary injury during this time interval..$^{15,26,31}$

Gait disturbance and imbalance are the most common ARE symptoms. For mildly to moderately symptomatic AREs, pharmacological treatments such as corticosteroid prescriptions are usually sufficient. However, 


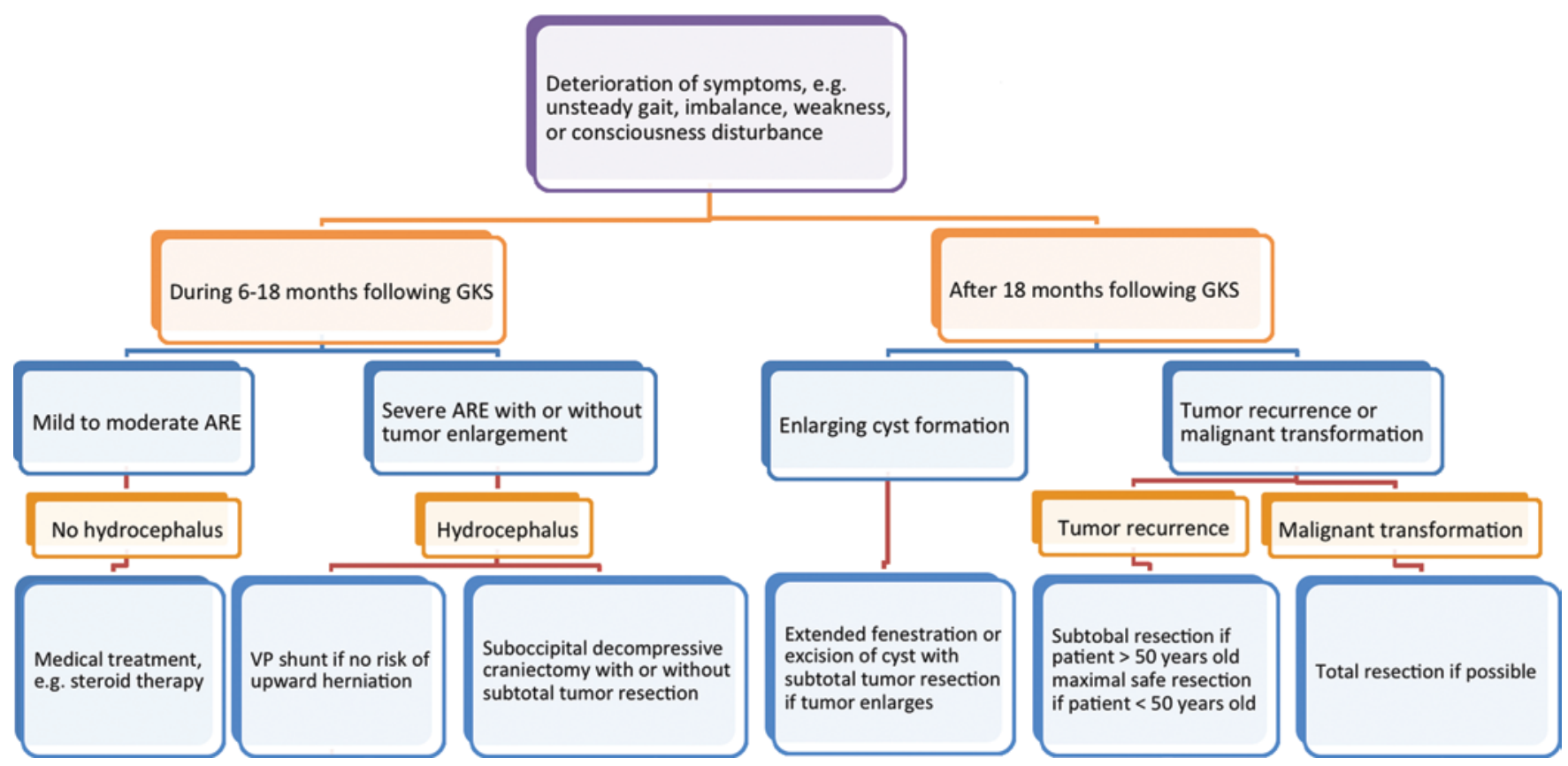

FIG. 3. Proposed algorithm for managing symptomatic VS after GKS.

in cases of severe ARE, symptoms may indicate worsening brainstem compression with or without obstructive hydrocephalus. Simple ventriculoperitoneal (VP) shunt placement is sufficient in those patients with obstructive hydrocephalus if the risk of upward herniation is low. However, patients in whom VP shunt placement failed or in those without obstructive hydrocephalus, suboccipital decompressive craniectomy may be indicated. In our series of 600 patients, a VP shunt was placed in 15 (2.5\%).

Tumor resection should be considered only when the mass effect is associated with tumor enlargement. Resection in these cases should be restricted to subtotal resection to decompress and to preserve cranial nerve function. Extensive skull base exposure as in the translabyrinthine approach may be necessary to gain wider exposure of the internal acoustic canal without excessive retraction on the swollen brain. Fortunately, because of improvements in imaging quality, dose planning, and in lowering margin doses, severe AREs in patients with VS are rare in modern radiosurgical series.

After radiosurgery, tumor recurrence must be carefully monitored in those with tumor expansion or enlargement. Compared with meningiomas and other benign brain tumors, VSs are more likely to initially expand after radiosurgery. ${ }^{40}$ Such expansion has been observed in $5 \%-62 \%$ of patients with VS in the early period after GKS. 7,21,32,36 Vestibular schwannomas also lose central contrast enhancement during this time period. Tumor regression is therefore typically observed after longer follow-up periods. Some authors reported that one-third of such enlarged tumors remained at the increased size without further growth, and only $2 \%$ of these tumors required additional intervention. ${ }^{34}$

The tumor expansion may be attributable to intratumoral hemorrhage, central necrosis, solid-part enlargement or cystic formation. Incidences of intratu- moral hemorrhage in VS after GKS are rare. ${ }^{17}$ We encountered intratumoral hemorrhage after GKS only in 3 patients $(0.5 \%)$. The hemorrhage may be the result of the natural history of tumor growth or a post-GKS effect on the tumor's vasculature. However, none of these 3 patients needed microsurgery for decompression, and all responded to conservative treatment. Hence, mild or chronic intratumoral hemorrhage alone should not be an indication for microsurgery. In our series, $19.9 \%$ of the patients showed transient tumor volume enlargement of $>$ $50 \%$ due to postradiosurgical swelling. Such initial tumor volume enlargement without neurological deterioration is not a surgical indication. Even in patients with symptomatic tumor swelling, these symptoms usually resolve after initiation of corticosteroid therapy. Microsurgery is rarely considered 6-18 months after radiosurgery, unless tumor recurrence or malignant transformation is suspected.

The distinction between transient tumor expansion and actual tumor recurrence may be difficult to ascertain, especially in the early post-GKS period. However, if rapid or progressive tumor enlargement is encountered several years after an initial period of stability after GKS, the possibility of recurrent tumor growth or malignant transformation should be considered. The time interval from the primary benign tumor to the malignant transformation ranges between 4 and 7 years. ${ }^{20}$ The incidence of malignant transformation of non-NF-2 VS may be low, but it was detected in some Group B patients after radiosurgery. Management strategies for recurrent tumors and tumors with malignant transformation, in our opinion, should differ from those for AREs.

The incidence of cyst formation in VS ranges from $4 \%$ to $15 \% .^{4,5,11,16}$ Cystic VS is believed to be associated with sudden expansion, a shorter clinical history $(<2$ years), atypical initial symptoms, and an increased rate of preoperative facial palsy. ${ }^{4,5,16}$ The mechanism of cyst 
formation is not yet fully understood; however, cyst expansion after radiosurgery for cystic VS is commonly observed. ${ }^{30}$ Possible mechanisms include intratumoral hemorrhage, ${ }^{29,42}$ osmotic changes from vascular damage, and extravasation of serum proteins into the extracellular matrix ${ }^{16,43}$ Cystic VS may enlarge more frequently than solid VS after GKS, and their volumes may also fluctuate after GKS. ${ }^{8}$ An important lesson may be learned from our case of cystic enlargement (Patient 11); microsurgery with extensive cyst resection or fenestration rather than simple aspiration or Ommaya reservoir placement was necessary for durable decompression and symptomatic relief.

\section{Strategies and Technical Aspects of Microsurgery for VS After GKS}

The goals of surgical resection after radiosurgery are the following: 1) obtain a tissue biopsy for disease diagnosis; 2) relieve the mass effect due to tumor, cyst, or both; 3) preserve cranial nerve and brainstem functions; and 4) decrease the risk of tumor recurrence. As mentioned above, if surgical decompression is considered for resolving any AREs during the early post-GKS period, subtotal tumor resection is usually sufficient for histological analysis, tumor control, and preservation of cranial nerve function. In contrast, maximal safe resection of VS is recommended in cases of sustained tumor enlargement, suspected recurrence, or malignant transformation after a period of initial stability.

Because of the grave prognosis of a median survival of $<1$ year in cases of malignant transformation, $, 212,13,18,25,34,35,39$ a more aggressive attitude should be taken toward the extent of VS resection. The tumor recurrence rate after complete microsurgical resection of benign VS has been reported to be as low as 0\%-3\%, whereas that after subtotal resection is believed to be approximately $20 \% .{ }^{23,27,32}$ In studies with small case numbers, some authors have reported that subtotal resection after radiosurgery resulted in a low tumor recurrence rate; however, analyses of tumor recurrence in large series with long-term follow-up are not yet available. ${ }^{32,36,37}$ It is reasonable to assume that tumor recurrence is directly related to the volume of residual viable tumor. Furthermore, reported recurrence rates may increase as the follow-up periods are extended. Taking these points into consideration, maximal safe resection, rather than subtotal resection, may benefit younger patients.

Some authors have suggested that because microsurgical resection is more difficult and aggressive after radiosurgery, microsurgery after radiosurgery may be associated with increases in postsurgical morbidities. ${ }^{20,32,36}$ Indeed, strong adherence of the tumor to the brainstem or other neurovascular structures is more commonly seen in patients with prior radiosurgical treatments. In addition, color changes may render the identification of cranial nerves more challenging. It is well recognized that radiosurgery devascularizes the tumor and makes the cranial nerves more vulnerable to ischemia during tumor dissection. Under such circumstances, postsurgical facial palsy usually occurs due to ischemic injury, despite anatomical preservation intraoperatively. We therefore agree with the consensus that total resection should not be recommend- ed in cases of tumor recurrence after GKS. However, with advanced skull base concepts and neuromonitoring techniques, maximal safe resection is feasible in this patient population.

For the past 4 years, we have used a different surgical approach, introduced a novel neuromonitoring method, and applied anatomical knowledge to improve the extent of resection and postsurgical outcomes. First, we followed the retrosigmoid approach with a near-presigmoid exposure. The transverse and sigmoid sinuses were skeletonized to lengths adequate for mobilization, resulting in a wider window with a more favorable trajectory to the brainstem. Using this approach, a fixed retractor on the brain is never employed, and thus retraction injury to the cranial nerves is minimized. Second, in addition to routine neuromonitoring of the facial and other lower cranial nerves, we have used the evoked blink reflex to help preserve trigeminal nerve function. The blink reflex is also useful when combined with free-run or triggered EMG for facial nerve function monitoring.

Third, after debulking the tumor, we meticulously identified the flocculus, choroid plexus, and internal meatus. The flocculus and choroid plexus are important landmarks directing us to the exit zone of the facial nerve, which is usually localized by repeated stimulation approximately $1 \mathrm{~cm}$ superiorly. Direct visualization of the nerve exit zone is difficult because of color changes and strong adherence to the tumor after GKS in most of our cases. The facial nerve may adhere to any quadrant of the tumor sphere. However, once the proximal and distal ends of the nerve are identified in the surgical field, the entire course of the nerve can be progressively delineated from its exit zone in 1 of the quadrants by repeated debulking and stimulation. The remaining part of the tumor can be then removed without difficulty.

The cranial nerve was never dissected away from the tumor capsule, unless the nerve was loosely attached to it. Meticulous dissection was kept within the tumor capsule, and the area suspected of surrounding the facial nerve was carefully trimmed. A thin cuff of tumor was left surrounding the involved cranial nerve (Fig. 1). In postoperative MRI follow-up scans, the near-absence of enhancement was usually mistaken as total resection. However, the residual tumor along with the facial nerve can be observed using the FIESTA sequences. Using these techniques, maximal tumor resections in 3 patients were achieved at our institution since 2009, and none of these patients experienced worsening of facial nerve function postsurgery.

In the current study, we emphasize the importance of the microsurgical techniques, neuromonitoring, and treatment strategies. Advancements in microsurgical and neuromonitoring techniques have given us more confidence in performing microsurgical resection of VS after GKS. In addition to the series we published in $2010,{ }^{19}$ here we added 6 more patients to the cohort that underwent this resection. We believe that the current study can offer readers insight into our experiences in treating VS after GKS and into the pitfalls that we encountered. As there will always be a cohort of patients who needs surgical management after GKS, we have striven to improve 
our radiosurgical and microsurgical techniques since our prior publication.

\section{Study Limitations}

This is a retrospective study with several limitations. The relatively small number of patients and the intermediate lengths of follow-up represent limitations and preclude substantial subgroup analyses. In particular, detection of complications arising from GKS-treated VS may require longer follow-up periods. Over the time period of the study, more advanced microsurgical and neuromonitoring techniques have improved the outcome of microsurgical resection of VS after GKS, which may represent an uncontrolled source of bias. Undoubtedly, a larger cohort with longer follow-up periods will provide more evidence and help us refine the management of VS after GKS.

\section{Conclusions}

Microsurgical resection for VS after radiosurgery remains challenging in terms of surgical indications, timing, or techniques: 1) The indications for surgical intervention in VS patients after GKS included AREs, tumor enlargement, and cyst development or enlargement. However, the most important factors for these interventions are the presence of symptoms and their severity. 2) Most VS patients with AREs can be treated with medications. The AREs may persist for as long as 18 months after GKS, but only 4 out of 600 patients $(0.7 \%)$ required a surgical intervention in our series. 3) Because of radiation effects, post-GKS tumors usually adhere to surrounding neurovascular structures. Therefore, we recommend maximal safe resection with comprehensive neuromonitoring, instead of total tumor resection.

\section{Disclosure}

The authors report no conflict of interest concerning the material or methods used in this study or the findings specified in this paper.

Author contributions to the study and manuscript preparation include the following. Conception and design: Hsu, Lee, Wu, Chung. Acquisition of data: Lee. Analysis and interpretation of data: Lee. Drafting the article: Lee, Chen. Critically revising the article: Lee, $\mathrm{Wu}$, Chen. Reviewed submitted version of manuscript: Lee, $\mathrm{Wu}$, Chung, Chen. Approved the final version of the manuscript on behalf of all authors: Hsu. Statistical analysis: Hsu, Lee. Administrative/technical/material support: Hsu, Lee, Wu, Chung, Pan. Study supervision: Hsu, Wu, Chung, Pan.

\section{References}

1. Baschnagel AM, Chen PY, Bojrab D, Pieper D, Kartush J, Didyuk O, et al: Hearing preservation in patients with vestibular schwannoma treated with Gamma Knife surgery. Clinical article. J Neurosurg 118:571-578, 2013

2. Best PV: Malignant triton tumour in the cerebellopontine angle. Report of a case. Acta Neuropathol 74:92-96, 1987

3. Carlson ML, Jacob JT, Pollock BE, Neff BA, Tombers NM, Driscoll CL, et al: Long-term hearing outcomes following stereotactic radiosurgery for vestibular schwannoma: patterns of hearing loss and variables influencing audiometric decline. Clinical article. J Neurosurg 118:579-587, 2013
4. Charabi S, Mantoni M, Tos M, Thomsen J: Cystic vestibular schwannomas: neuroimaging and growth rate. J Laryngol Otol 108:375-379, 1994

5. Charabi S, Tos M, Thomsen J, Rygaard J, Fundova P, Charabi B: Cystic vestibular schwannoma-clinical and experimental studies. Acta Otolaryngol Suppl 543:11-13, 2000

6. Chopra R, Kondziolka D, Niranjan A, Lunsford LD, Flickinger JC: Long-term follow-up of acoustic schwannoma radiosurgery with marginal tumor doses of 12 to 13 Gy. Int J Radiat Oncol Biol Phys 68:845-851, 2007

7. Chung WY, Liu KD, Shiau CY, Wu HM, Wang LW, Guo WY, et al: Gamma knife surgery for vestibular schwannoma: 10year experience of 195 cases. J Neurosurg 102 Suppl:87-96, 2005

8. de Ipolyi AR, Yang I, Buckley A, Barbaro NM, Cheung SW, Parsa AT: Fluctuating response of a cystic vestibular schwannoma to radiosurgery: case report. Neurosurgery 62:E1164E1165, 2008

9. Foote KD, Friedman WA, Buatti JM, Meeks SL, Bova FJ, Kubilis PS: Analysis of risk factors associated with radiosurgery for vestibular schwannoma. J Neurosurg 95:440-449, 2001

10. Friedman WA, Bradshaw P, Myers A, Bova FJ: Linear accelerator radiosurgery for vestibular schwannomas. J Neurosurg 105:657-661, 2006

11. Fundová P, Charabi S, Tos M, Thomsen J: Cystic vestibular schwannoma: surgical outcome. J Laryngol Otol 114:935939, 2000

12. Han DH, Kim DG, Chi JG, Park SH, Jung HW, Kim YG: Malignant triton tumor of the acoustic nerve. Case report. J Neurosurg 76:874-877, 1992

13. Hanabusa K, Morikawa A, Murata T, Taki W: Acoustic neuroma with malignant transformation. Case report. J Neurosurg 95:518-521, 2001

14. Hasegawa T, Kida Y, Kato T, lizuka H, Kuramitsu S, Yamamoto T: Long-term safety and efficacy of stereotactic radiosurgery for vestibular schwannomas: evaluation of 440 patients more than 10 years after treatment with Gamma Knife surgery. Clinical article. J Neurosurg 118:557-565, 2013

15. Hasegawa T, Kida Y, Yoshimoto M, Koike J, Goto K: Evaluation of tumor expansion after stereotactic radiosurgery in patients harboring vestibular schwannomas. Neurosurgery 58:1119-1128, 2006

16. Kameyama S, Tanaka R, Kawaguchi T, Fukuda M, Oyanagi K: Cystic acoustic neurinomas: studies of 14 cases. Acta Neurochir (Wien) 138:695-699, 1996

17. Karampelas I, Alberico RA, Plunkett RJ, Fenstermaker RA: Intratumoral hemorrhage after remote subtotal microsurgical resection and gamma knife radiosurgery for vestibular schwannoma. Acta Neurochir (Wien) 149:313-317, 2007

18. Kudo M, Matsumoto M, Terao H: Malignant nerve sheath tumor of acoustic nerve. Arch Pathol Lab Med 107:293-297, 1983

19. Lau T, Olivera R, Miller T Jr, Downes K, Danner C, van Loveren HR, et al: Paradoxical trends in the management of vestibular schwannoma in the United States. Clinical article. J Neurosurg 117:514-519, 2012

20. Lee CC, Yen YS, Pan DH, Chung WY, Wu HM, Guo WY, et al: Delayed microsurgery for vestibular schwannoma after gamma knife radiosurgery. J Neurooncol 98:203-212, 2010

21. Linskey ME, Lunsford LD, Flickinger JC: Neuroimaging of acoustic nerve sheath tumors after stereotaxic radiosurgery. AJNR Am J Neuroradiol 12:1165-1175, 1991

22. Litvack ZN, Norén G, Chougule PB, Zheng Z: Preservation of functional hearing after gamma knife surgery for vestibular schwannoma. Neurosurg Focus 14(5):E3, 2003

23. Lownie SP, Drake CG: Radical intracapsular removal of acoustic neurinomas. Long-term follow-up review of 11 patients. J Neurosurg 74:422-425, 1991

24. Massager N, Nissim O, Delbrouck C, Delpierre I, Devriendt 
D, Desmedt F, et al: Irradiation of cochlear structures during vestibular schwannoma radiosurgery and associated hearing outcome. J Neurosurg 107:733-739, 2007

25. McLean CA, Laidlaw JD, Brownbill DS, Gonzales MF: Recurrence of acoustic neurilemoma as a malignant spindle-cell neoplasm. Case report. J Neurosurg 73:946-950, 1990

26. Nagano O, Higuchi Y, Serizawa T, Ono J, Matsuda S, Yamakami I, et al: Transient expansion of vestibular schwannoma following stereotactic radiosurgery. Clinical article. J Neurosurg 109:811-816, 2008

27. Ojemann RG: Management of acoustic neuromas (vestibular schwannomas) (honored guest presentation). Clin Neurosurg 40:498-535, 1993

28. Pan DH, Guo WY, Chung WY, Shiau CY, Chang YC, Wang LW: Gamma knife radiosurgery as a single treatment modality for large cerebral arteriovenous malformations. J Neurosurg 93 Suppl 3:113-119, 2000

29. Park CK, Kim DC, Park SH, Kim JE, Paek SH, Kim DG, et al: Microhemorrhage, a possible mechanism for cyst formation in vestibular schwannomas. J Neurosurg 105:576-580, 2006

30. Pendl G, Ganz JC, Kitz K, Eustacchio S: Acoustic neurinomas with macrocysts treated with Gamma Knife radiosurgery. Stereotact Funct Neurosurg 66 (Suppl 1):103-111, 1996

31. Pollock BE: Management of vestibular schwannomas that enlarge after stereotactic radiosurgery: treatment recommendations based on a 15 year experience. Neurosurgery 58:241248, 2006

32. Pollock BE, Lunsford LD, Kondziolka D, Sekula R, Subach BR, Foote RL, et al: Vestibular schwannoma management. Part II. Failed radiosurgery and the role of delayed microsurgery. J Neurosurg 89:949-955, 1998

33. Régis J, Delsanti C, Roche PH, Thomassin JM, Pellet W: [Functional outcomes of radiosurgical treatment of vestibular schwannomas: 1000 successive cases and review of the literature.] Neurochirurgie 50:301-311, 2004 (Fr)

34. Scheithauer BW, Erdogan S, Rodriguez FJ, Burger PC, Woodruff JM, Kros JM, et al: Malignant peripheral nerve sheath tumors of cranial nerves and intracranial contents: a clinicopathologic study of 17 cases. Am J Surg Pathol 33:325-338, 2009
35. Shin M, Ueki K, Kurita H, Kirino T: Malignant transformation of a vestibular schwannoma after gamma knife radiosurgery. Lancet 360:309-310, 2002

36. Shuto T, Inomori S, Matsunaga S, Fujino H: Microsurgery for vestibular schwannoma after gamma knife radiosurgery. Acta Neurochir (Wien) 150:229-234, 2008

37. Slattery WH III, Brackmann DE: Results of surgery following stereotactic irradiation for acoustic neuromas. Am J Otol 16:315-321, 1995

38. Snell JW, Sheehan J, Stroila M, Steiner L: Assessment of imaging studies used with radiosurgery: a volumetric algorithm and an estimation of its error. Technical note. J Neurosurg 104:157-162, 2006

39. Son EI, Kim IM, Kim SP: Vestibular schwannoma with malignant transformation: a case report. J Korean Med Sci 16: 817-821, 2001

40. Spiegelmann R, Lidar Z, Gofman J, Alezra D, Hadani M, Pfeffer R: Linear accelerator radiosurgery for vestibular schwannoma. J Neurosurg 94:7-13, 2001

41. Sun S, Liu A: Long-term follow-up studies of Gamma Knife surgery with a low margin dose for vestibular schwannoma. Clinical article. J Neurosurg 117 Suppl:57-62, 2012

42. Suzuki H, Toyoda S, Muramatsu M, Shimizu T, Kojima T, Taki W: Spontaneous haemorrhage into metastatic brain tumours after stereotactic radiosurgery using a linear accelerator. J Neurol Neurosurg Psychiatry 74:908-912, 2003

43. Unger F, Walch C, Papaefthymiou G, Feichtinger K, Trummer M, Pendl G: Radiosurgery of residual and recurrent vestibular schwannomas. Acta Neurochir (Wien) 144:671-677, 2002

\footnotetext{
Manuscript submitted June 28, 2014.

Accepted August 8, 2014.

Please include this information when citing this paper: DOI: 10.3171/2014.8.GKS141312.

Address correspondence to: Sanford P. C. Hsu, M.D., Department of Neurosurgery, Neurological Institute, Taipei Veterans General Hospital, 17F, 201 Shipai Rd., Sec. 2, Beitou, Taipei 11217, Taiwan, R.O.C.email: doc6623g@gmail.com.
} 